\title{
Modification of surface-state dispersion upon Xe adsorption: A scanning tunneling microscope study
}

\author{
Ji-Yong Park, U. D. Ham, S.-J. Kahng, and Y. Kuk* \\ Department of Physics and Center for Science in Nanometer Scale, Seoul National University, Seoul, 151-742, Korea \\ K. Miyake, ${ }^{1}$ K. Hata, ${ }^{1}$ and H. Shigekawa ${ }^{1,2}$ \\ ${ }^{1}$ Institute of Applied Physics and CREST, Japan Science and Technology Corporation (JST), University of Tsukuba, \\ Tsukuba, 305-8573, Japan \\ ${ }^{2}$ Department of Chemistry and Biotechnology, Graduate School of Engineering, The University of Tokyo, Hongo, \\ Tokyo, 113-8656, Japan \\ (Received 24 October 2000)
}

\begin{abstract}
The modification of the surface electronic structure by an adsorbate is measured quantitatively with scanning tunneling microscopy and spectroscopy for the first time. The standing wave of the $\mathrm{Cu}$ surface-state electrons is utilized to probe the subtle change in the electronic structure on the Xe-covered $\mathrm{Cu}(111)$. The observed Fermi wavelength on the Xe-covered surface is longer by $15 \%$ than on the bare $\mathrm{Cu}$ surface. The change upon $\mathrm{Xe}$ adsorption is explained with the observed modified dispersion of the $\mathrm{Cu}$ surface state; upward shift by $(130 \pm 20) \mathrm{meV}$ with almost same effective mass.
\end{abstract}

There has been interest recently in the surface state electrons, localized on the (111) surfaces of noble metals and the (0001), (1010) surfaces of Be. ${ }^{1-3}$ The electrons are known to play as important a role as the bulk electrons in surfacerelated phenomena, such as adsorption, surface magnetism, surface and step energetics, and catalysis. ${ }^{4}$ They reveal nearly two-dimensional (2D) characteristics with a nearly free-electron-like dispersion. ${ }^{5}$ The electrons in the surface states, therefore, form standing waves of quantum interference on the surfaces when there is a potential well or a barrier, created by a point defect or a line defect ${ }^{1,2}$ or even a quantum corral. ${ }^{6}$ This long range modulation of local density of states (LDOS) is explained with many electron effect like Friedel oscillation, change in the electronic state density near the Fermi level by electron screening of the defect potential. Direct imaging in real space of the modulation has been possible only with a scanning tunneling microscope (STM). ${ }^{1,2}$ Since the observation of the electron confinement in nanometer-scale structures, ${ }^{6-8}$ understandings in several surface-related phenomena, such as the shape of Fermi contour, ${ }^{3,9,10}$ surface state dispersions, ${ }^{1,2,11}$ surface state lifetime, ${ }^{12,13}$ and surface Kondo temperature, ${ }^{14}$ have been greatly advanced.

It is well known that adsorption of foreign atoms or molecules modifies the geometric structure, the work function, and the surface electronic state of a clean surface. In many cases, the adsorption removes the surface electronic state and leaves the bulk electronic state. In the case of alkali metals and rare gases, however, their adsorptions modify the surface electronic structure so slightly that the surface states are persistent. ${ }^{4}$ The changes of the surface states and their dispersions with adsorbates have been often studied with photoemission spectroscopy, including two photon photoemission spectroscopy (2PPES). Though the surface state dispersions on clean surfaces have been successfully measured with STM and scanning tunneling spectroscopy (STS), influence of the adsorbates on the surface states has not been tried with STM. It has been believed that the surface state electrons may not tunnel through the adsorbate layer.

In this Rapid Communication, we report a way to quantitatively measure the change of the surface electronic state by an adsorbate on a metal surface with STM and STS for the first time. We measure the standing waves of the surface state electrons not only on the bare $\mathrm{Cu}(111)$ surface but also on the surface with one monolayer (ML) Xe. The small change in the Fermi wavelength (15\%) and the modified dispersion relation of the surface state measured by STS are used to explain the proposed redistribution of the charge density with the adsorption of Xe. ${ }^{15}$

Our experiments are performed in a low temperature $\mathrm{STM}^{16}$ at the base pressure of $<1 \times 10^{-10}$ torr. It works at the temperature down to $\sim 5 \mathrm{~K}$. A Cu(111) single crystal is cleaned by repeated cycles of $\mathrm{Ar}^{+}$ion sputtering and annealing up to $900 \mathrm{~K}$. High purity Xe gas is dynamically dosed through a precision leak valve and a stainless steel tube with the diameter of $1 / 16$ inch to the cold sample surface. This dynamic supply ensures proper partial pressure of Xe gas around the sample surface, but not in the whole STM chamber. The sample can be annealed by controlling the liquid helium supply or using a heater attached on the back of the sample.

$\mathrm{Xe}$ atoms nucleate at step edges and defect sites on the $\mathrm{Cu}$ terrace at the coverage of $\sim 0.7 \mathrm{ML}$ dosed at $10 \mathrm{~K}$. The Xe layer can be agglomerated by annealing at $>30 \mathrm{~K}$ (Ref. 17) and a flat Xe layer of $1 \mathrm{ML}$ height can be obtained. The surface is, then, partially $(\sim 70 \%)$ covered with $1 \mathrm{ML}$ high Xe patches as shown in Fig. 1(a). Bright small, hexagonal islands are Xe islands of $2 \mathrm{ML}$ height, which are pinned around defects even after annealing. Using the $\mathrm{Cu}$ lattice image as in Fig. 1(b), the length scale can be self-calibrated exactly. Figure 1(c) is a high-resolution image of the $\mathrm{Xe}$ layer, showing the perfect crystallinity. The nearest neighbor distance of the Xe adlayer is determined to be (4.25 


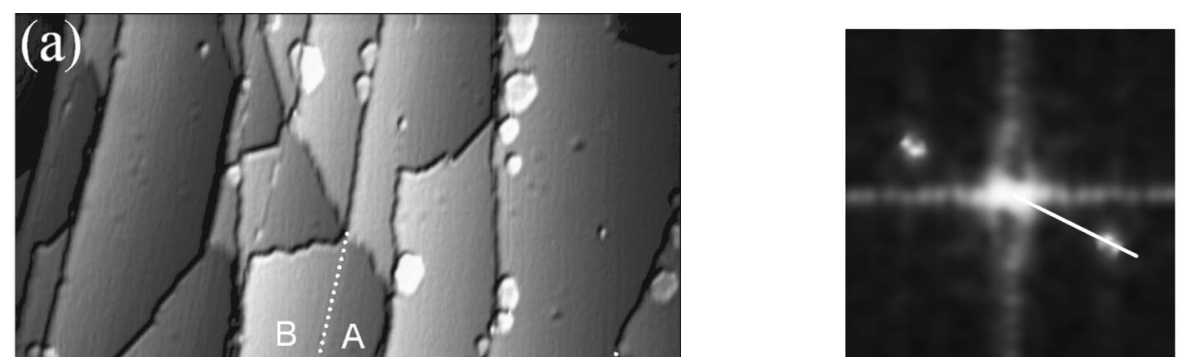

(a)

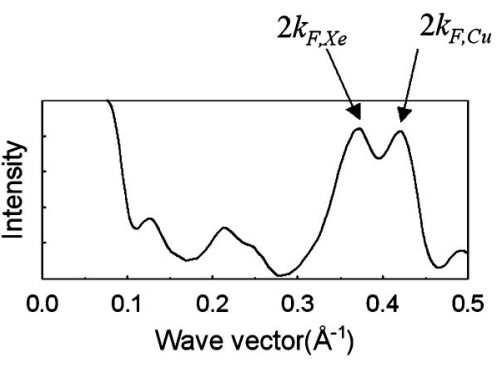

(b)

FIG. 2. (a) A Fourier-transformed (logarithm of the power spectrum) image of pair correlation of Fig. 1(d) $\left(1.2 \AA^{-1} \times 1.2 \AA^{-1}\right)$. Two peaks at either side originate from different wavelengths of the standing waves on the Xe-covered and the bare $\mathrm{Cu}$ surface. (b) Cross section along white line in (a). A difference of $\sim 15 \%$ is visible. $k_{F, \mathrm{Xe}}$ and $k_{F, \mathrm{Cu}}$ denote the Fermi wave vectors for the $1 \mathrm{ML}$ $\mathrm{Xe}$-covered and the bare $\mathrm{Cu}(111)$, respectively.

ingly, we observe the standing wave patterns even on the Xe-covered surface. With the step-flow growth of the Xe layer at the lower step edge at high temperature, ${ }^{17}$ standing waves are imaged both on the 1 ML Xe-covered surface on the lower terrace and on the bare $\mathrm{Cu}$ surface on the upper terrace simultaneously at the region, marked by a square in Fig. 1(a), as shown in Fig. 1(d). With this image, any possibility of artifacts due to the tip change can be excluded. The slight difference in height is due to the difference in LDOS on the Xe-covered and the bare $\mathrm{Cu}$ surface at the bias voltage. The white protrusions along the boundary are $\mathrm{Xe}$ atoms pinned at upper step edges of $\mathrm{Cu}$ during annealing process. The wavelength of the standing waves on Xe layer is longer by $15 \%$ than that on $\mathrm{Cu}$ surface.

The periodic structures can be revealed more clearly if the image is presented in the Fourier space. This technique was already applied to determine the Fermi contour with the electron standing waves. ${ }^{9,10}$ In order to visualize the two different periodicities, we take the Fourier transformation (FT) of a positional pair-correlation function, in which the positional pair-correlation function, $G(\mathbf{r})$, is defined as

$$
G(\mathbf{r})=\int h\left(\mathbf{r}^{\prime}\right) \cdot h\left(\mathbf{r}^{\prime}+\mathbf{r}\right) d \mathbf{r}^{\prime},
$$

where $h(\mathbf{r})$ is the height in STM image at position $\mathbf{r}$. Figure 2(a) is an example of the FT of the pair-correlation. Two peaks are visible at both sides, confirming the existence of two standing waves with different wavelengths; those on the $\mathrm{Xe}$-covered and the bare $\mathrm{Cu}$ surface. The features at the center and along the axis are artifacts that are originated from noise and insufficient sampling at small wave vectors. Figure 2(b) is the cross section along the line from center through these peaks, revealing two peaks with the instrumental and the thermal broadening. The wavelength on the Xe-covered surface is again found to be $15 \%$ longer than that on the bare $\mathrm{Cu}$ surface as deduced from this cross section.

At first sight, it is difficult to imagine how the standing waves can be observed on the Xe-covered surface, because (i) the Xe layer does not have a surface state near the Fermi level, (ii) the wavelength is too large to be the atomic corrugation of $\mathrm{Xe}$ (atomic radius $=4.3 \AA$ ). In the case of $\mathrm{Xe}$ atoms,

the highest occupied atomic orbital, $5 p$, and the lowest un-

surface state electrons are observed near defects or steps on clean $\mathrm{Cu}(111)$ surface at $5 \mathrm{~K}$ as reported earlier. ${ }^{1}$ Surpris- 

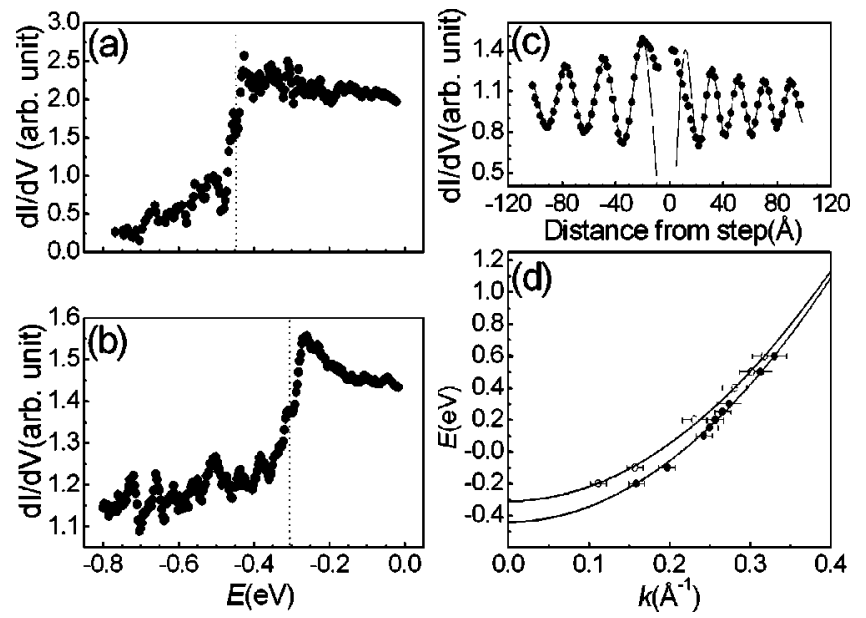

FIG. 3. Experimental $d I / d V$ spectra taken from (a) $\mathrm{Cu}(111)$ and (b) $1 \mathrm{ML}$ Xe-covered $\mathrm{Cu}(111)$ at $5 \mathrm{~K}$. (c) $d I / d V$ plot perpendicular to a step at $E=-200 \mathrm{meV}$ (solid circle). The line is a fit by Eq. (2). Left side (negative distance) is the Xe-covered surface and right side (positive distance) is the bare $\mathrm{Cu}$ surface. (d) Dispersion relation of the clean (solid circle) and Xe-covered (open circle) $\mathrm{Cu}(111)$ surface state at $5 \mathrm{~K}$. The solid lines are quadratic fits to data.

occupied atomic orbital, $6 s$, are far from the Fermi level. ${ }^{15}$ But according to a local density approximation calculation, ${ }^{15}$ the Fermi-level conduction electrons extend further out into the vacuum in the presence of Xe adsorbates than on clean metal surface. Previous experiments also suggested the pile-up of the conduction electron density at the Fermi level in the core of adsorbed rare gas atoms ${ }^{18}$ and reduced tunneling barrier of electrons through the adsorbed Xe layer. ${ }^{19} \mathrm{We}$ conclude that the observed standing waves are caused by electrons in the $\mathrm{Cu}$ surface state of which the charge density extends through $1 \mathrm{ML}$ Xe layer with a longer decay length than on the bare surface. No sign of standing waves can be found on 2 ML Xe patches such as bright islands in Fig. 1(a), indicating that the surface state electrons on $\mathrm{Cu}$ surface can contribute to the tunneling process only through the $1 \mathrm{ML}$ Xe.

There can be several mechanisms, which can result in longer wavelengths on Xe-covered layer. (i) The confinement of the surface state electrons on a narrow terrace can result in different wavelength in the STM image. ${ }^{7,8}$ As our experimental results are obtained on wide terraces $(>200 \AA)$, the confinement effect can be neglected. (ii) It was shown that the bulk electrons also contribute to the formation of the standing waves with different wavelength. ${ }^{10}$ But this contribution near the step was found to be negligible ${ }^{10}$ and our results do not support this possibility since most of our images are obtained near steps. (iii) The longer wavelength may be explained with the modified dispersion of the surface state with $1 \mathrm{ML}$ Xe. In order to verify this scenario, we perform STS on both the Xe-covered and the bare $\mathrm{Cu}$ surface. The measured $d I / d V$ versus sample voltage dependence at one point is approximately proportional to the product of LDOS and the tunneling probability at the bias. It is obtained under open feedback condition with a lock-in amplifier. By measuring the local $d I / d V$ spectra on both surfaces, the onsets of surface states can be determined as in Figs. 3(a) and 3(b). Well-known onset of the surface state at $(440 \pm 10) \mathrm{meV}$ below Fermi level is visible on clean $\mathrm{Cu}$ surface in Fig. 3(a).
On the $\mathrm{Cu}$ surface with $1 \mathrm{ML} \mathrm{Xe}$, the onset is shifted toward Fermi level by $(130 \pm 20) \mathrm{meV}$ as shown in Fig. 3(b). A shift of $\sim 100 \mathrm{meV}$ was obtained in a 2 PPES experiment. ${ }^{20}$ In the case of alkali adsorbates, they change the surface electronic structure such that lowering of the work function and downward shift of the surface state are expected. ${ }^{21}$ Although the work function is lowered on $1 \mathrm{ML} \mathrm{Xe}$ on $\mathrm{Cu}(111),{ }^{20}$ the shift is upward, i.e., opposite direction. The $d I / d V$ images were acquired simultaneously with constant current image. The dispersion of the surface states can also be determined by analyzing $d I / d V$ images at varying bias voltages. We fit the $d I / d V$ line scans perpendicular to a step by LDOS of the $2 \mathrm{D}$ nearly free electrons of effective mass $m^{*}$ when they are scattered off the one-dimensional barrier (step). The LDOS, $\rho_{s}$, is given by

$$
\rho_{s}=L_{0}\left[1-r J_{0}\left(2 k_{0} x\right)\right]
$$

where $J_{0}$ is the zero-order Bessel function, $L_{0}=m^{*} /\left(\pi \hbar^{2}\right)$, $x$ is the distance from the step, $k^{2}=2 m^{*} E / \hbar^{2}$, and $r$ is the reflectance of the step. Figure 3(c) is one example of such a fit at $E=-200 \mathrm{meV}$. The results are summarized in Fig. 3(d) on the $\mathrm{Xe}$-covered and the bare $\mathrm{Cu}$ surfaces. Since the $d I / d V$ image represents the convolution of the topography and the LDOS, the topographic contribution can be minimized by taking the image at a large tunneling gap. ${ }^{22}$ The measured dispersion on the bare $\mathrm{Cu}$ surface is similar to the one reported earlier. ${ }^{1}$ Again it is confirmed that the dispersion of $\mathrm{Cu}$ surface with $1 \mathrm{ML} \mathrm{Xe}$ is shifted by $(130 \pm 20) \mathrm{meV}$ and the effective electron mass is hardly changed within the parabolic band approximation $\left[(0.40 \pm 0.02) m_{e}\right.$ and $(0.42$ $\left.\pm 0.03) m_{e}\right]$. The change of other characteristics can be deduced from our data. For example, the onset of the surface state is broadened on the Xe-covered surface [compare Figs. $3(\mathrm{a})$ and $3(\mathrm{~b})$ ], suggesting the reduced lifetime of the surface state, ${ }^{12}$ possibly due to the opening of a new decay channel resulting from phonons on Xe overlayer. We find that the shift of the surface state persists on 1 ML Xe islands as small as $20 \mathrm{~nm}^{2}$, discriminating the effect of impurity.

Our results clearly demonstrate that significant parts of the signal from adsorbate-covered surface in STM can come from the substrate through the adsorbates, especially which do not have much DOS near the Fermi level. We also believe that our findings can be extended to other adsorbate systems, such as alkali metals. Our result can be utilized such that the surface state may be intentionally modified with rare gas atoms to investigate whether the depopulation or population of the surface state influences growth behaviors and others, ${ }^{23}$ which is in controversy recently. ${ }^{24}$

In summary, we show that the effect of adsorbate on surface state can be successfully investigated with STM for the first time. The surface state electrons of $\mathrm{Cu}$ near Fermi level are still visible with the presence of $1 \mathrm{ML}$ Xe layer due to the redistribution of charge density in such a way that it extends further at Xe adsorbates. It is believed that the longer wavelength of standing waves on the Xe-covered surface compared with the bare $\mathrm{Cu}$ surface is due to the shift of surface state dispersion of $\mathrm{Cu}$ with $\mathrm{Xe}$ adsorption.

After submission of this paper, we realize similar experiments are in progress on the alkali-covered noble metal surfaces. $^{25}$ 
The authors would like to thank N. D. Lang, J. Ihm, and J. $\mathrm{Yu}$ for carefully reading this manuscript. This work was partially supported by Korean Ministry of Science and Technology through Creative Research Initiative Program and Ko- rean Ministry of Education, BSRI-98-2416. Support from Grant-in-Aid for Scientific Research from the Ministry of Education, Science, Sport and Culture of Japan is also acknowledged.
*To whom correspondence should be addressed; Electronic address: ykuk@phya.snu.ac.kr

${ }^{1}$ M. F. Crommie, C. P. Lutz, and D. M. Eigler, Nature (London) 363, 524 (1993).

${ }^{2}$ Y. Hasegawa and Ph. Avouris, Phys. Rev. Lett. 71, 1071 (1993); $\mathrm{Ph}$. Avouris and I.-W. Lyo, Science 264, 942 (1994).

${ }^{3}$ Ph. Hofmann, B. G. Briner, M. Doering, H.-P. Rust, E. W. Plummer, and A. M. Bradshaw, Phys. Rev. Lett. 79, 265 (1997); B. G. Briner, Ph. Hofmann, M. Doering, H.-P. Rust, E. W. Plummer, and A. M. Bradshaw, Phys. Rev. B 58, 13931 (1998).

${ }^{4}$ See, S. D. Kevan and W. Eberhardt, in Angle-Resolved Photoemission, edited by S. D. Kevan (Elsevier, Amsterdam, 1992); E. Bertel, Phys. Status Solidi A 159, 235 (1997); N. Garciá and P. A. Serena, Surf. Sci. 330, L665 (1995).

${ }^{5}$ Electronic Surface and Interface States on Metallic Systems, edited by E. Bertel and M. Donath (World Scientific, Singapore, 1995).

${ }^{6}$ M. F. Crommie, C. P. Lutz, and D. M. Eigler, Science 262, 218 (1993).

${ }^{7}$ L. Bürgi, O. Jeandupeux, A. Hirstein, H. Brune, and K. Kern, Phys. Rev. Lett. 81, 5370 (1998).

${ }^{8}$ J. Li, W. Schneider, R. Berndt, and S. Crampin, Phys. Rev. Lett. 80, 3332 (1998).

${ }^{9}$ L. Petersen et al., Phys. Rev. B 57, R6858 (1998).

${ }^{10}$ L. Petersen, P. Laitenberger, E. Lægsgaard, and F. Besenbacher, Phys. Rev. B 58, 7361 (1998).

${ }^{11}$ T. Yokoyama, M. Okamoto, and K. Takayanagi, Phys. Rev. Lett. 81, 3423 (1998).
${ }^{12}$ J. Li, W. Schneider, R. Berndt, O. R. Bryant, and S. Crampin, Phys. Rev. Lett. 81, 4464 (1998).

${ }^{13}$ L. Bürgi, O. Jeandupeux, H. Brune, and K. Kern, Phys. Rev. Lett. 82, 4516 (1999); O. Jeandupeux, L. Bürgi, A. Hirstein, H. Brune, and K. Kern, Phys. Rev. B 59, 15926 (1999).

${ }^{14}$ M. F. Crommie (private communication).

${ }^{15}$ D. M. Eigler, P. S. Weiss, E. K. Schweizer, and N. D. Lang, Phys. Rev. Lett. 66, 1189 (1991).

${ }^{16}$ Ji-Yong Park, Ph.D. thesis, Seoul National University, 2000.

${ }^{17}$ Ji-Yong Park, S.-J. Kahng, U. D. Ham, Y. Kuk, K. Miyake, K. Hata, and H. Shigekawa, Phys. Rev. B 60, 16934 (1999).

${ }^{18}$ D. M. Eigler and S. Schultz, Phys. Rev. Lett. 54, 1185 (1985).

${ }^{19}$ G. Ehrlich and F. G. Hudda, J. Chem. Phys. 30, 493 (1959).

${ }^{20}$ M. Wolf, E. Knoesel, and T. Hertel, Phys. Rev. B 54, R5295 (1996).

${ }^{21}$ S. A. Lindgren and L. Walldèn, Solid State Commun. 28, 283 (1978); 34, 671 (1980); S. D. Kevan, Surf. Sci. 178, 229 (1986).

${ }^{22}$ J. Li, W. Schneider, and R. Berndt, Phys. Rev. B 56, 7656 (1997).

${ }^{23}$ N. Memmel and E. Bertel, Phys. Rev. Lett. 75, 485 (1995); E. Bertel, P. Roos, and J. Lehmann, Phys. Rev. B 52, R14 384 (1995); M. Giesen, G. Schulze Icking-Konert, and H. Ibach, Phys. Rev. Lett. 80, 552 (1998); 82, 3101 (1999).

${ }^{24}$ K. Morgenstern, G. Rosenfeld, G. Comsa, E. Lægsgaard, and F. Besenbacher, Phys. Rev. Lett. 85, 468 (2000); M. Giesen and H. Ibach, ibid. 85, 469 (2000).

${ }^{25}$ Talk by J. Kliewer and R. Berndt in SPS-2000, Hamburg, Germany (2000). 